\title{
Lumen
}

Selected Proceedings from the Canadian Society for Eighteenth-Century Studies

\section{La mythologie de la mine : la fiction anti-économique chez le marquis de Sade}

\section{Richard Spavin}

Volume 34, 2015

URI : https://id.erudit.org/iderudit/1028512ar

DOI : https://doi.org/10.7202/1028512ar

Aller au sommaire du numéro

Éditeur(s)

Canadian Society for Eighteenth-Century Studies / Société canadienne d'étude du dix-huitième siècle

ISSN

1209-3696 (imprimé)

1927-8284 (numérique)

Découvrir la revue

Citer cet article

Spavin, R. (2015). La mythologie de la mine : la fiction anti-économique chez le marquis de Sade. Lumen, 34, 71-92. https://doi.org/10.7202/1028512ar 


\title{
La mythologie de la mine : la fiction anti-économique chez le marquis de Sade
}

\author{
RiCHARD SPAVIN \\ University of Toronto
}

Dans un des rares ouvrages que Sade signe de son nom («par D.A.F. Sade, auteur d'Aline et Valcour»), le recueil de nouvelles Les crimes de l'amour, il inclut un «conte suédois », Ernestine, narré en grande partie au fond d'une mine - une des fameuses mines de cuivre de Falun, au nord-ouest de Stockholm. Dans les carrières de cet espace souterrain, qui font office de «rues", se trouvent non sans fantastique des «maisons, des temples, des auberges, des travaux, de la police et même des juges $^{1} »$. La réalité historique du lieu s'oppose à une riche intertextualité qui évoque d'autres espaces souterrains - qu'on songe à l'Enfer de Dante, au Tartare, lieu de punition dans la mythologie grecque ou bien aux topoï terrifiants du mélodrame ${ }^{2}$. Or la mine dans Ernestine s'en démarque : elle se veut moins une «descente aux enfers » qu'une conversion du vice à la vertu. Une telle réécriture de la mine permet à Sade d'élaborer une histoire héroïque qui souligne non seulement les terreurs de l'univers commercial d'en haut, soit l'emprise économique du métal qui domine partout ${ }^{3}$, mais qui transforme son lieu de prédilection (usine à monnaie) en un espace de gloire, dont les acteurs peuvent remonter à la surface et reconquérir l'imaginaire des lecteurs.

1. Ernestine, dans Euvres complètes, édité par Jean-Jacques Pauvert, Paris, Société Nouvelles des Éditions Pauvert, 1988, t. 10, p. 377.

2. Voir Maria Walecka-Garbalinska, «La mine suédoise comme espace mélodramatique», dans Simone Bernard-Griffiths et Jean Sgard (éd.), Mélodrames et romans noirs, 1750-189o, Toulouse, Presses universitaires du Mirail, 2000, p. 277-298.

3. Jean Sgard, «Prévost et le romanesque suédois», dans Gunnar von Proschwitz (éd.), Influences. Relations culturelles entre la France et la Suède, Göteborg, Société royale des sciences et des belles-lettres de Göteborg, 1988, p. 99. 
En effet, Sade ne figure généralement pas dans l'histoire des idéologies commerciales. Il est absent, avec juste raison, du livre d'Albert Hirschman, Les passions et les intérêts, qui explique si bien l'essor de l'intérêt particulier, du désir de la richesse avant le «triomphe» du capitalisme. Le marquis réagit différemment que ses prédécesseurs au changement de paradigme que subit son siècle, où, dès le classicisme, on assiste à la «démolition » du héros et à l'exaltation de la productivité, des valeurs marchandes et du gain ${ }^{4}$. Chez Sade, l'« ordre libertin » des écrits pornographiques non signés pourrait cependant exprimer à cet égard un pendant sexuel, plutôt radicalisé, de l'intérêt particulier. Une certaine «économie » des corps violentés - où la jouissance est calculée, comptabilisée et industrialisée - se prête admirablement à l'études. Mais quelle vision donne-t-il plus précisément de cet «effondrement» de la gloire qui nous amènera à représenter l'homme en dehors de toute rêverie moralisatrice, «tel qu'il l'est vraiment ${ }^{6}$ » ? Si les rouages qui sapent les idéaux de la volonté générale et de l'héroïsme sont en branle durant la seconde moitié du XVIII siècle, ils sont pourtant moins inexorables que ce qu'on pourrait le croire. La «gloire» de Napoléon, synthèse de valeurs républicaines et absolutistes du tournant des Lumières, ne couvait pas évidemment en vase clos?. Elle se retrouve notamment chez ce contemporain inattendu qu'est le marquis de Sade, et ce, sous la forme d'une curieuse mythologie qui intègre, en les inversant, les forces de l'économie et leur empire sur les passions.

\section{L'héroïsme et l'économie de la gloire}

La mine de cuivre sert de décor narratif pour une nouvelle que le marquis décrit lui-même, dans le sous-titre du recueil des Crimes de l'amour, comme étant «tragique et héroïque». Du côté tragique, l'intrigue de Ernestine ne fait pas exception, ne lésinant aucunement sur les malheurs qui accablent le sort des personnages les plus vertueux et

4. Voir Paul Bénichou, Morales du Grand Siècle, Paris, Gallimard, 1948.

5. Voir Hénaff, Sade, l'invention du corps libertin, Paris, PUF, 1978; D. Martyn, Sublime Failures: The Ethics of Kant and Sade, Détroit, Wayne State University Press, 2003, p. 29-50.

6. A. Hirschman, The Passions and the Interests: Political Arguments for Capitalism Before its Triumph, Princeton (N.J.), Princeton University Press, 1977, p. 9-12.

7. Voir l'ouvrage de R. Morrissey, Napoléon et l'héritage de la gloire, Paris, PUF, 2010. 
pour lesquels la mine constitue une certaine rétribution. Le personnage d'Oxtiern, un des deux criminels du récit, doit y purger une peine de prison à perpétuité pour avoir commis des crimes «sans exemple» qui font l'objet de l'histoire enchâssée racontée par le guide Falkeneim. Celui-ci conte les mésaventures d'une jeune amoureuse, Ernestine, qui veut épouser Herman, son amant, qui travaille en tant que caissier chez la veuve Scholtz qui s'oppose à l'union. Cette dernière a des vues sur Herman et lui propose un mariage qu'elle justifie comme une transaction ou en termes de rétribution: Herman doit l'épouser en gage de reconnaissance pour avoir fait fructifier ses investissements. Oxtiern arrive ensuite sur la scène pour séduire Ernestine et déposséder Herman de sa bien-aimée. Lorsque les deux amants refusent de renoncer à leur amour, on décide de se venger d'eux: Herman est victime d'un coup monté par la Scholtz; on le pend à Stockholm, jugé coupable d'avoir volé les coffres de la veuve, tandis qu'Ernestine est assassinée (accidentellement) par son propre père, dans un complot ourdi par Oxtiern. Après que la totalité des horreurs est révélée au roi Gustave, la Scholtz est pendue à Stockholm et Oxtiern est banni aux mines, là où narrateur le rencontre.

À bien des égards, la mine d'Ernestine semble entrer dans une histoire des décors souterrains qui rappelle les espaces narratifs du roman noir et du mélodrame ${ }^{8}$. Bien que constituant des lieux naturels qui attirent d'abord la curiosité des scientifiques - les articles et les passages sur la métallurgie dans l'Encyclopédie et l'Histoire naturelle des minéraux de Buffon sont abondants -, les mines se prêtent admirablement aux ouvrages de fiction qui les tirent vers l'imaginaire gothique'. Typiquement, le rôle d'un tel décor n'est pas d'inspirer la vertu héroïque à ses lecteurs, mais plutôt le contraire. Les mines sont des endroits qui inspirent la frayeur, qui servent d'espace carcéral, où la brutalité de l'espace déteint sur les sensibilités des prisonniers qui y sont condamnés - sophisme pathétique oblige. On peut se reporter à

8. Voir le récent article de Michel Delon sur le rôle métaphorique de la mine dans la scénographie théâtrale et romanesque de l'époque, "Les Entrailles de la terre”. Métaphore de la mine et imaginaire du souterrain (1750-1815) », dans Par les siècles et par les genres. Mélanges en l'honneur de Giorgetto Giorgi, Paris, Classiques Garnier, 2014, p. 259-272.

9. Voir Joël Castonguay-Bélanger, «Histoire naturelle et surnaturelle : les profondeurs gothiques de la mine de sel», dans Catriona Seth (éd.), Imaginaires gothiques. Aux sources du roman noir français, Paris, Desjonquères, 2010, p. 84-97. 
la description que donne Jean-François Regnaud d'une mine dans son Voyage de Suède (1731). Regnaud, voyageur mais aussi homme de théâtre, relate sa visite de Kopparberget dans des termes plutôt dramatiques qui font appel davantage au sentiment qu'à la fonction industrielle de l'espace souterrain :

Mais descendons dans cet abîme pour en mieux concevoir l'horreur. On nous conduisit d'abord dans une chambre où nous changeâmes d'habits, et prîmes chacun un bâton ferré pour nous soutenir dans les endroits les plus dangereux. De là nous entrâmes dans la mine par une bouche d'une longueur et d'une profondeur épouvantable [...]. Nous descendîmes dans ce fond par quantité de degrés qui y conduisaient. [...] Nous descendîmes plus de deux lieues dans la terre par des chemins épouvantables, tantôt sur des échelles tremblantes, tantôt sur des planches légères, et toujours dans de continuelles appréhensions ${ }^{10}$.

Regnard structure son texte verticalement, en répétant les mêmes mots descriptifs. Le danger s'accroît à mesure qu'on descend dans les «entrailles de la terre». Jean Sgard l'a déjà mis en évidence: en essayant d'écrire une relation de voyage, Regnard finit par écrire une descente plutôt romanesque dans la mine qui équivaut à la descente aux Enfers ${ }^{11}$. D'où la fascination de Prévost qui développe son «aventure intéressante des mines de Suède » à partir des observations du voyageur, récit fondé sur l'attraction «gravitationnelle» du crime ${ }^{12}$. Une tendance se met définitivement en place: la mine permet de sonder les profondeurs du mal; elle représente un espace punitif, aux antipodes de la gloire qui récompenserait les belles et grandes actions héroïques.

Quant au marquis, loin de renoncer aux poncifs thématiques du roman gothique (le crime, le viol, le meurtre), il rejette néanmoins l'épouvante que la mine évoque traditionnellement pour les écrivains de l'époque. À l'inverse de Regnard et de Prévost, il découvre dans ce

10. Voyage de Regnard en Flandre, en Hollande, en Danemark et en Suède (1681), éd. par A. de Marsy, 1874, cité par Maria Walecka-Garbalinska, «La mine suédoise », art. cit., p. 281 .

11. Jean Sgard, «Prévost et le romanesque suédois », art. cit., p. 95.

12. L'héroïne du conte de Prévost refuse de se séparer de son mari qui est condamné à vivre dans la mine pour ses crimes. Elle décide de le rejoindre en sautant symbolique dans la mine, un trou «aussi profond qu'inégal et ténébreux » (Prévost, «Aventures des mines de Suède », Euvres, t. XXV, Genève, Slatkine Reprints, 1969, p. 10). 
décor souterrain une civilisation exemplaire, à l'abri de tout «danger », qui situe nécessairement le mal ailleurs:

À l'aide d'un panier et d'une corde, machine disposée de façon à ce que le trajet se fasse sans aucun danger, nous arrivâmes au fond de cette mine, et nous nous trouvâmes en un instant à cent vingt toises de la surface du sol. Ce ne fut pas sans étonnement que je vis, là, des rues, des maisons, des temples, des auberges, du mouvement, des travaux, de la police, des juges, tout ce que peut offrir enfin le bourg le plus civilisé de l'Europe.

Après avoir parcouru ces habitations singulières, nous entrâmes dans une taverne, où Falkeneim obtint de l'hôte tout ce qu'il fallait pour se rafraîchir, d'assez bonne bière, du poisson sec, et une sorte de pain suédois, fort en usage à la campagne, fait avec les écorces du sapin et du bouleau, mêlées à de la paille, à quelques racines sauvages, et pétries avec de la farine d'avoine; en faut-il plus pour satisfaire au véritable besoin? Le philosophe qui court le monde pour s'instruire, doit s'accommoder de toutes les mœurs, de toutes les religions, de tous les temps, de tous les climats, de tous les lits, de toutes les nourritures, et laisser au voluptueux indolent de la capitale ses préjugés... son luxe... ce luxe indécent qui, ne contentant jamais les besoins réels, en crée chaque jour de factices aux dépens de la fortune et de la santéér.

Sade intègre le décor gothique pour en inverser les connotations. Un voyage dans les entrailles de la Terre se présente ici comme un éloignement du mal, dorénavant identifié au « luxe indécent », c'est-àdire ce cercle vicieux qui fait des appétits matériels une nécessité quasi existentielle. Dans la mine, on est effectivement à l'abri des tentations qui poussent au crime et qui régulent, semblerait-il, les structures économiques du monde d'en haut. Moins un appel au pathos, la mine comporte une fonction thétique plus générale qu'il faut inscrire dans le projet romanesque de l'auteur.

Quant à l'«héroïsme» du conte, deuxième versant du projet des Crimes de l'amour, il convient de rappeler les définitions théoriques que formule l'auteur dans son Idée sur les romans qui sert de préface au recueil de nouvelles. Selon lui, le roman moderne se charge de représenter les «héros», de les «immortaliser», afin de véhiculer une certaine «vertu» à la postéritée ${ }^{14}$. La mise en récit de l'héroïsme

13. Ernestine, op. cit., p. 376-377.

14. Idée sur les romans, dans Euvres complètes, op. cit., p. 62-63. 
possède une drôle de généalogie qui nous confronte à nos origines narratives, initialement « déraisonnables », à savoir des fictions qui ont pour motifs «la crainte, l'espérance, et le dérèglement d'esprit» qui immortalisent non des «héros » mais des «Dieux» :

N'en doutons point: ce fut dans les contrées qui, les premières, reconnurent des Dieux, que les romans prirent leur source, et par conséquent en Égypte, berceau certain de tous les cultes; à peine les hommes eurent-ils soupçonné des êtres immortels, qu'ils les firent agir et parler; dès lors, voilà des métamorphoses, des fables, des paraboles; en un mot, voilà des ouvrages de fictions, dès que la fiction s'empare de l'esprit des hommes. Voilà des livres fabuleux, dès qu'il est question de chimères: quand les peuples, d'abord guidés par des prêtres, après s'être égorgés pour leurs fantastiques divinités, s'arment enfin pour leur roi ou pour leur patrie, l'hommage offert à l'héroïsme balance celui de la superstition, non seulement on met, très sagement alors, les héros à la place des Dieux, mais on chante les enfants de Mars comme on avait célébré ceux du ciel; on ajoute aux grandes actions de leur vie, ou las de s'entretenir d'eux, on crée des personnages qui leur ressemblent... qui les surpassent, et bientôt de nouveaux romans paraissent, plus vraisemblables sans doute, et bien plus faits pour l'homme que ceux qui n'ont célébré que des fantômes ${ }^{15}$.

Un tel extrait explique bien les ambiguïtés et les contradictions qui cernent la représentation de l'héroïsme à la fin du XvıII siècle. Le héros s'oppose à ce qui fonde, en fait, ses stratégies narratives et rhétoriques. Dans une logique de supplantation, l'homme-conteur arrive à se libérer des fantômes de la divinité en les remplaçant progressivement par des histoires non moins imaginaires de la libération civile et politique. Aussi le roman moderne emprunte-t-il les mêmes armes du storytelling religieux, précisément parce qu'il lui succède, à la manière d'une rétorsion formelle, qui consiste à reprendre l'argumentation de l'adversaire en montrant qu'elle s'applique en réalité contre lui ${ }^{16}$.

La conception sadienne de l'héroïsme possède une rhétorique dérivative qui est fondée sur le «balance[ment]» des artifices qui contredisent les vertus civiles et politiques et sur le remplacement de ceux-ci par des fables qui chantent les «libérateurs d'une nation ${ }^{17}$.

15. Idem.

16. Olivier Reboul, Introduction à la rhétorique, Paris, PUF, 2005 [1991], p. 175.

17. Idée sur les romans, op. cit., p. 63. 
En d'autres termes, le héros, construction romanesque, s'avère un adversaire habile et astucieux; sa résistance se formule par palimpseste, à l'écoute de ce qu'elle vise à subvertir. Sur ce point, Robert Morrissey explique dans son ouvrage Napoléon et l'héritage de la gloire la particularité de la situation à la fin du XVIII ${ }^{\mathrm{e}}$ siècle, lorsque l'idéologie économique et commerciale tend à discréditer l'exemplarité héroïque et la gloire qui lui sert de récompense. La pensée libérale, que ce soit les abeilles de Mandeville ou la main invisible de Smith, éloigne les intérêts et les passions de la conscience de la collectivité, réduisant la volonté générale à un résultat qui se réalise à notre insu. Chez Montesquieu, en effet, l'honneur, principe de la monarchie, est dépossédé de son héroïsme aristocratique et guerrier, associé plutôt à l'ambition et à l'intérêt personnel. Cela sape les caractères définitoires du héros, c'est-à-dire son désintéressement personnel et sa parfaite identification à la collectivitél ${ }^{8}$. R. Morrissey cite à ce sujet l'ouvrage d'un jeune Jean-Paul Marat, Les chaînes de l'esclavage, publié d'abord en anglais en 1771, puis en français en 1793, qu'il écrit lors d'un séjour en Angleterre. Louvrage singularise le luxe et l'opulence, qui préparent l'arrivée du despotisme, en leur opposant des vertus qui nous permettent de conserver notre liberté: l'activité, la frugalité, le désintéressement, la vigilance et l'amour de la gloire ${ }^{19}$. Enfin, si la gloire à la fin du XVIII siècle, ainsi que le théorise R. Morrissey, dresse une opposition entre la vertu et l'intérêt, elle n'est en aucune manière une suppression de l'un des deux termes; elle constitue plutôt un «troisième terme» qui permet, d'une part, de «tirer parti d'un ensemble complexe de motivations individuelles plus ou moins intéressées ${ }^{20}$ » et, de l'autre, «d'élaborer un ensemble de pratiques tendant à gommer la différence entre amour de la gloire et amour du gain, entre reconnaissance et rémunération, entre dévouement à la patrie et souci de soi $^{21}{ }^{2}$. L'élégante notion d'une «économie de la gloire », titre de son chapitre sur Napoléon, rappelle l'idéologie héroïque du romanesque sadien, où l'écrivain, chargé de créer de nouvelles fictions, intègre, en l'inversant, précisément ce que le héros cherche à déboulonner.

18. Robert Morrissey, Napoléon et l'héritage de la gloire, op. cit., p. 75.

19. Jean-Paul Marat, Les chaînes de l'esclavage, cité par Robert Morissey, Napoléon et l'héritage de la gloire, op. cit., p. 78.

20. Robert Morissey, Napoléon et l'héritage de la gloire, op. cit., p. 79.

21. Ibid., p. 107. 
Dans le cas d'Ernestine, il s'agira d'expliquer comment la main de l'auteur traduit une nouvelle forme de despotisme qui inscrit Sade dans le combat séculaire entre la vertu et le commerce, dilemme auquel il apporte un certain remède en supplantant la domination de l'argent, l'emprise économique du métal, par une drôle de «mine», grandement fictive et fabuleuse, qui transforme les intérêts personnels en des passions publiques. En identifiant la dialectique qui s'installe entre les éléments historiques et fictifs du conte, la mine se posera, contrairement à sa fonction économique et industrielle au tournant du siècle, comme un espace proprement héroïque.

\section{La réalité versus la fiction}

Dans son «Catalogue raisonné des CEuvres de M. de $\mathrm{S}^{* * * * *}$ », Sade se prononce sur les différentes nouvelles des Crimes de l'amour, où Ernestine se démarque non seulement du fait qu'il la juge la «meilleure du recueil, relativement à la conduite et au dénouement ${ }^{22}$ », mais en raison de son «auctorialité » que Sade accorde au personnage qui visite lui-même les lieux: «l'auteur est, écrit-il, lui-même en action et est un des personnages de l'estampe». Les descriptions des autres nouvelles, comme Juliette et Raunai et Laurence et Antonio, commentent davantage l'intervention du marquis dans la composition si bien qu'on peut se poser plus de questions sur la nature de ce personnage et son voyage en Suède.

En effet, Ernestine présente de nombreux éléments spatiotemporels qui renforcent une prétention narrative à la vérité. Le narrateur se rend lui-même en Suède, nous faisant part des différentes étapes de son voyage, dont l'épisode suédois représente l'escale la plus significative: «Ce fut dans cette intention que je partis de Paris le 20 juillet 1774, et, après avoir traversé la Hollande, la Westphalie, et le Danemark, j’arrivai en Suède vers le milieu de l'année suivante ${ }^{23}$.» Ce voyage fictif en Suède peut se comparer avec le voyage réel que fait Gabriel Jars (le frère de celui qui publie ses Voyages métallurgiques) en 1766 pour visiter les mines les plus importantes du nord de l'Europe: «D'abord Hollande, Hanovre, montagnes du Hartz (4 mois), Saxe, Mansfeld,

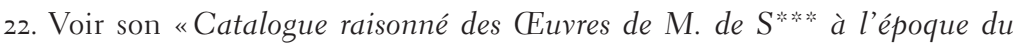
$1^{e r}$ octobre 1788 », cité par Pauvert, dans Sade, Euvres complètes, op. cit., p. 46.

23. Ernestine, op. cit., p. 375. 
Hambourg, Copenhague, Kongsberg en Norvège, Suède ${ }^{24}$. » Si Daniel Roche a déjà mis en évidence la contribution de cet ouvrage à la reconnaissance d'une «naissance d'une économie industrielle ${ }^{25}$ », les frères Jars (toute la famille Jars ${ }^{26}$ ) se sont montrés dévoués à l'essor technologique et économique du royaume français, efforts que l'idéologie industrielle pouvait caractériser comme héroïques et glorieux à tel point que Gabriel Jars, le cadet, se nomme « un élève de l'État ${ }^{27}$ ». Le voyage dans le nord de l'Europe, dont la Suède fut une destination plutôt symbolique pour fraterniser avec le roi, avait pour but « d'observer les progrès de l'industrie $\&$ de l'esprit humain » afin de « rapport[er] à sa patrie les fruits de leur progrès \& et du moins quelques-uns des avantages que l'étranger a su se procurer ${ }^{28}$.

Or le narrateur sadien voyage précisément afin d'«admirer, dit-il, ce peuple sage, vertueux, sobre et magnanime, qu'on peut appeler le modèle du Nord ${ }^{29}$ » Une sorte de voyage spirituel et non technologique l'amène dans les mines presque par accident ou par «curiosité» littéraire:

24. Gabriel Jars, Voyages métallurgiques ou recherches et observations sur les mines et forges de fer, la fabrication de l'acier, celle du fer-blanc, E plusieurs mines de charbon de terre, faites depuis l'année 1757 jusques et compris 1769, en Allemagne, Suède, Norvège, Angleterre ๒ Écosse, Lyon, Regnault, 1774, p. xxiv.

25. Daniel Roche, Humeurs vagabondes. De la circulation des hommes et de l'utilité des voyages, Paris, Fayard, 2003, p. 306; voir aussi Isabelle Laboulais, «Les Voyages métallurgiques de Gabriel Jars (1774-1781). Un recueil au service de l'art de l'exploitation des mines », dans Pierre-Yves Beaurepaire et Pierrick Pourchasse (éd.), Les circulations internationales en Europe, années 1680-années 1780, Rennes, Presses universitaires de Rennes, 2010, p. 181-195.

26. Une curiosité de la famille Jars était effectivement d'associer le nom «Gabriel Jars » à la métallurgie: les deux frères que nous évoquons ici se nomment tous deux «Gabriel Jars» tout comme leur père et leur grand-père qui travaillent tous dans l'industrie minière (voir Isabelle Laboulais, «Les Voyages métallurgiques », art. cit., p. 181$)$.

27. Dans L'éloge de M. Jars, prononcé à l'Académie des Sciences de Paris, le 25 Avril 1770, on peut lire les lignes suivantes: "La vue des mines, des travaux \& des établissements nécessaires à leur exploitation, le rendirent métallurgiste, \& bientôt il fallut modérer cette ardeur, \& l'empêcher de passer la plus grande partie de son temps dans les souterrains; l'envie de s'instruire lui faisait oublier le danger auquel il exposait sa vie \& sa santé; cette espèce de phénomène parvient jusqu’aux oreilles de feu M. Vallières, à son passage à Lyon, il voulut voir le jeune homme, \& en fut si content, qu'il jugea nécessaire de l'envoyer à la Capitale pour y cultiver des talents si marqués \& si précieux; \& dès ce moment, il devient en quelque sorte l'élève de l'État » (dans Voyages métallurgiques, op. cit., p. xxi-xxii).

28. Voyages métallurgiques, op. cit., p. xix-xx.

29. Ernestine, op. cit., p. 375. 
Au bout d'un séjour de trois mois à Stockholm mon premier objet de curiosité, dit-il, se porta sur ces fameuses mines, dont j’avais tant lu de descriptions et dans lesquelles j'imaginais rencontrer peut-être quelques aventures semblables à celles que nous rapporte l'abbé Prévost, dans le premier volume de ses anecdotes; j’y réussis... mais quelle différence ${ }^{30}$ !

Le récit de la «différence » à la première personne, véritable motif du récit de voyage, aspire à un effet d'objectivité, issu de la tradition sceptique $^{31}$. Le degré de curiosité est garant de crédibilité, comme en témoigne le manque de «ressemblance» d'avec ses voyages précédents, par exemple, aux «catacombes de Rome ou de Naples ${ }^{32}$. De plus, l'intrigue se réfère à des espaces qui existent réellement et qui gagnent en concrétude par les références temporelles: tout se passe durant le règne de Gustave III, après le coup d'État - la conspiration des «bonnets »- de 1772. Le conte, paru en 1800, est presque d'actualité et le narrateur intra-diégétique fait tout pour faire croire à son lecteur qu'il y est réellement, présent pour entendre les noms des «personnages » dont il va jusqu'à préciser la prononciation ${ }^{33}$. Le voyage industriel qui avait pour but de rapporter des connaissances concrètes de l'étranger est supplanté ici par un voyage moralisateur qui perpétue de vieux stéréotypes sur l'héroïsme suédois.

Ainsi, au fond des mines, une intéressante relation se met-elle en place entre l'invraisemblable du souterrain et le vraisemblable du monde d'en haut. Le lecteur est invité à considérer une juxtaposition entre les effets d'objectivité des embrayeurs spatiotemporels et le flagrant fantastique des mines, où la civilité du décor, soit une taverne dans laquelle on peut boire $\mathrm{d}^{\prime}$ "assez bonne bière ${ }^{34}$ ", présente plus d'invraisemblances que les représentations du roman gothique. Si Sade laisse la fiction s'emparer de la représentation de la mine, devenue une sorte d'utopie, il s'efforce de décrire d'autres éléments géographiques et historiques d'une manière plus conforme à la réalité. En répondant à ce décalage, nous avancerons qu'au lieu de renforcer les références

30. Ibid., p. 376 .

31. Voir sur ce sujet l'article d'Isabelle Moreau, «Lectures libertine de la matière viatique », Études de lettres, 3, 2006, p. 9-24.

32. Ernestine, op. cit., p. 376.

33. «Il est essentiel de prévenir que toutes les lettres se prononcent dans les noms du Nord, que l'on ne dit point négligemment: Herman, Sanders, Scholtz, mais qu'il faut dire comme s'il y avait Herman-e, Sander-ce, Scholt-ce, etc. (ibid., p. 381).

34. Ibid., p. 377. 
au contexte historique en particulier, la mine se détache de son ancrage spatiotemporel pour infléchir la fiction au vu d'un certain argument, énonciation profondément morale qui n'est pas relative uniquement à la Suède mais qui donne une forme narrative au dilemme idéologique entre le commerce et la vertu qui pèse sur les esprits du tournant du XVIII ${ }^{\mathrm{e}}$ siècle $^{35}$. Il n'est pas surprenant que Sade, écrivain-prisonnier, choisisse d'étudier la question de l'économie sous le prisme de la justice criminelle.

\section{La punition rétributive versus la punition corrective}

Ainsi que la lecture de Jean-Baptiste Jeangène Vilmer l'a mis en évidence, la représentation de la justice comporte une partie intégrante de l'argumentation du conte suédois et participe de ce qu'il appelle plus généralement le «moralisme» sadien. On peut effectivement remarquer la qualité critique des nombreuses expressions «rétributives» de la justice, surtout celles qui condamnent Oxtiern et sa complice la Scholtz à leurs punitions respectives. Si celui-là est banni aux mines, celle-ci est pendue à Stockholm, "payant [ainsi] l'horreur de ses forfaits, sur le même échafaud où elle avait fait mourir l'innocent ${ }^{36}{ }^{3}$. Sade décrit les punitions comme des paiements, des échanges quasi monétaires qui ont pour intention de rétablir la vertu mais qui ne font que perpétuer notre conception appauvrie de la justice et du système pénal ${ }^{37}$. C'est pourquoi le bannissement d'Oxtiern dans les mines, où il sera mis au travail en une sorte de punition utilitaire, s'oppose à la fin du conte à sa libération, héroïque et sacrificielle, par le colonel Sanders. Ce dernier finit par accepter le remords du criminel mais en l'astreignant à expier ses crimes par des actes vertueux afin de rendre à la société ce qu'il a enlevé. La courte dissertation de Sanders vers la fin de la nouvelle plaide contre l'inutilité morale de l'enfermement:

Vos maux, d'ailleurs, réparent-ils les miens? puis-je être heureux de vos douleurs? votre détention acquitte-t-elle le sang que vos barbaries ont fait répandre? Je serais aussi cruel que vous... aussi injuste, si je le

35. Voir J. G. A. Pocock, Virtue, Commerce, History: Essays on Political Thought and History, Chiefly in the Eighteenth Century, New York / Cambridge, Cambridge University Press, 1985 .

36. Ernestine, op. cit., p. 427.

37. Jeangène Vilmer, Sade moraliste, Genève, Droz, 2005, p. 449. 
pensais. La prison dédommage-t-elle la société des maux qu'il lui a faits? ... Il faut le rendre libre, cet homme, si l'on veut qu'il répare, et, dans ce cas, il n'en est aucun qui ne le fasse, il n'en est pas un seul qui ne préfère le bien à l'obligation de vivre dans les fers. Ce que peut inventer sur cela le despotisme, chez quelques nations, ou la rigueur des lois, chez d'autres, le cœur de l'honnête homme le désavoue.... ${ }^{38}$

Sanders, porte-parole de l'auteur, commente aigrement l'équilibre que le système pénal se contente d'établir entre la victime et le criminel, transférant les «maux » de celui-là à celui-ci, en un échange plutôt morbide et cruel. Le vieux militaire semble renoncer au sentiment de vengeance - plaisir de la victime de voir souffrir le malfaiteur - pour améliorer la société qu'il avait mise à mal, sacrifiant ses propres sentiments de violence au profit de la collectivité. Lorsque les deux personnages sortent de la mine, le lecteur comprend pourtant que le sacrifice «moral» de Sanders est en réalité un prétexte pour libérer le criminel de la mine et le traduire en justice lui-même dans le combat:

Sénateur, dit-il à Oxtiern, il s’agit maintenant de me faire raison; j'espère que vous êtes assez brave pour ne pas me refuser, et que vous avez assez d'esprit pour être convaincu que le plus puissant motif qui m'ait fait agir dans ce que je viens de faire, était l'espoir de me couper la gorge avec vous $^{39}$.

Sade insère une tension entre deux formes d'héroïsme, deux formes de sacrifice. Dans l'échange initial, fait au bénéfice des «spectateurs », l'héroïsme sert une cause plus grande que les maux de la victime: le bien collectif l'emporte sur la rancune. En sortant de la mine, pourtant, Sanders se contredit. Le sacrifice du duel satisfait dorénavant un désir de revanche pour retomber dans une philosophie négative de la justice. La réflexivité du verbe «se couper la gorge » insiste sur le cercle vicieux dans lequel est enfermée la justice rétributive qui décide du sort des victimes, payant la souffrance par la souffrance.

Oxtiern, lui, en est tout de même attendri et choisit de mettre fin au duel, se disant trop indigne pour se battre contre l'honorable Sanders. Le sacrifice devient celui du criminel qui s'élance sur l'épée du colonel, lui donnant le «sang» qu'il est venu réclamer. Cet acte héroïque rétablit celui que Sanders prétendait vouloir faire dans la

38. Ernestine, op. cit., p. 429 .

39. Ibid., p. 430. 
mine. Satisfait du sacrifice du criminel, ce dernier répète la même dissertation, maintenant en toute sincérité, lui demandant à nouveau de jouir de la liberté qu'il lui rend et de tâcher de réparer "par quelques belles actions» tous les crimes qu’il avait perpétrés ${ }^{40}$. En effet, la fin du conte raconte une véritable conversion du criminel en personnage vertueux. Sade n'est pas ironique dans sa représentation de la vertu; nous sommes ainsi d'accord avec Jeangène Vilmer: Ernestine n'est pas une glorification insidieuse ou détournée du crime ${ }^{41}$, mais plutôt une occasion de réfléchir sur la morale d'une manière indirecte et «fractale» qui reconstruit une voie vers la vertu ${ }^{42}$. Toutefois, la fonction critique de la nouvelle est, dans son ensemble, plus portée sur le commerce que sur la justice, plus soucieuse de contrecarrer l'influence «despotique» que le commerce fait subir à la dynamique sociale. En lisant le conte dans cette optique, on comprend mieux pourquoi la justice, réglée de manière rétributive, est présentée comme une corruption économique, voire le résultat d'un embourgeoisement complet de la société qui s'oppose aux origines militaires et héroïques de la nation, incarnées par Charles XII et d'autres figures annoncées dès la première page de la nouvelle: Alaric, Attila, Théodoric ${ }^{43}$. En d'autres termes, c'est l'identité nationale de la Suède qui est en cause, en proie au commerce, aux appétits rapaces, qui vicie toutes les strates de la société.

40. Ibid., p. 431.

41. Dans la belle préface que Michel Delon écrit pour Les crimes de l'amour, le relief que le marquis ajoute à ses personnages aurait pour effet d'approfondir l'envergure du crime, lui donnant un «frémissement» et une «ambiguïté » qui n'existent pas dans les scènes de torture et d'orgies des écrits clandestins ( Préface », Les crimes de l'amour, Paris, Gallimard, 1987, p. 10-11).

42. Jeangène Vilmer, Sade moraliste, op. cit., p. 457.

43. Jean Sgard voit dans l'évocation de ces trois figures la préfiguration du mal: «l'esprit du mal souffle ici (en Suède) avec une particulière brutalité. C'est pourquoi, aux images classiques de la folie héroïque et tragique, incarnées par Christine de Suède ou Charles XII, [Sade] a mêlé celle d'Alaric, d'Attila et de Théodoric, fléaux de Dieu dont Oxtiern est le lointain descendant» («Prévost et le romanesque suédois ", art. cit., p. 99). Pour notre part, nous tenons à mentionner que ces figures ne sont pas «suédoises» et que la contamination étrangère fait précisément partie de la problématique de la nouvelle. Nous y reviendrons. 


\section{L'absolutisme commercial : Nordköping, la Scholtz, la mine}

Nordköping, "ville absolument de commerce ${ }^{44}$ ", renvoie à une nouvelle forme d'«absolutisme», à un despotisme commercial, dont la Scholtz se révèle le chef incontesté. Personnage métonymique, la Scholtz incarne la puissance que le commerce exerce plus généralement sur la société: « une femme comme Mme Scholtz, à la tête de l'une des plus riches maisons de la Suède, devait tenir le premier rang $^{45}$ ». Et ce, sans qu'il y ait de limitation morale à son « règne». Sa puissance est précisément machiavélique, divorcée des origines vertueuses et naturelles du pouvoir souverain, expliquant l'opposition entre elle et Sanders, que son influence risque d'« écraser » :

Il s'en fallait bien que le colonel Sanders, peu à son aise et retiré du service, eût autant de prépondérance dans Nordkoping que la veuve Scholtz; la considération de celle-ci s'étendait fort long, pendant que l'autre, déjà oublié, n'était plus vu, parmi des hommes qui, en Suède comme partout, n'estiment les gens qu'en raison de leur faveur ou de leur richesse, n'était plus regardé, dis-je, que comme un simple particulier que le crédit et l'or pouvaient facilement écraser, et Mme Scholtz, comme toutes les âmes basses, avait eu bientôt fait ce calcul ${ }^{46}$.

Le pouvoir de la richesse et son influence sur l'opinion publique témoignent de la désuétude de la gloire militaire, devise désormais «insolvable». Plus loin, lorsque le riche Oxtiern se joint à la veuve, le narrateur décrit leur alliance à l'instar d'une «ligue» qui se dresse contre Herman, comme si un véritable pouvoir absolu ravageait Nordköping. Le lexique du constitutionnalisme renforce l'hypallage commercial-politique; les fameux «freins» qui limitent le pouvoir monarchique n'ont aucun effet sur les passions d'Oxtiern qui s'était déjà montré résistant au souverain, chef légitime, dans la conspiration des «bonnets » où s'affrontent le roi Gustave et ses féodaux:

Le comte, fort riche, et maître depuis fort peu de toute sa fortune, ne soupçonnait aucune borne à ses fougueux désirs; tout ce que la raison ou les circonstances pouvaient leur apporter d'obstacles ne devenait qu'un aliment de plus à leur impétuosité; sans principes comme sans vertu, encore imbu des préjugés d'un corps dont l'orgueil venait de

44. Ernestine, op. cit., p. $3^{84}$.

45. Ibid., p. 384 .

46. Ibid., p. 387 . 
lutter contre le souverain même, Oxtiern s'imaginait que rien au monde ne pouvait imposer de frein à ses passions ${ }^{47}$.

La souveraineté se révèle être compromise par les forces économiques et aristocratiques qui, s'unissant contre la vertu, usurpent le pouvoir. Il y existe une situation sociopolitique étrange, une sorte de «mercantilisme intériorisé », déjà présent chez Rousseau, qui traduit une vision antagonique des relations étrangères à l'intérieur du pays lui-même; la volonté d'écraser et d'engloutir des pays rivaux par les richesses métalliques - le «bullionisme» - est effectivement internalisée. Cette dégénérescence historique est particulièrement bien exemplifiée par les mines, annoncées comme étant la propriété des financiers anglais, dès le début de la nouvelle:

Ces mines, longtemps la plus grande ressource de l'État, tombèrent bientôt dans la dépendance des Anglais, à cause des dettes contractées par les propriétaires avec cette nation, toujours prête à servir ceux qu'elle imagine pouvoir engloutir un jour, après avoir dérangé leur commerce ou flétri leur puissance, au moyen de ses prêts usuraires ${ }^{48}$.

Les mines, qui servent de lieu de punition, sont non seulement soumises à une commercialisation de la société, mais elles sont également asservies à une domination géopolitique où les dettes et les ressources monétaires remplacent les guerres de conquête. Cette description de la mine fait ainsi référence à la grandeur de l'État pour ensuite «tomb $[\mathrm{er}]$ » entre les mains vénales des Anglais. La conquête de ces derniers - qui prépare leur engloutissement sous le vernis d'un «service»- préfigure les relations de confiance qu'instaurent les criminels de l'histoire envers leurs victimes; victimes qu'ils prétendent eux aussi «servir» pour en fait les dominer et les «écraser». Cela est particulièrement frappant dans les échanges entre le comte Oxtiern et Sanders au bal, où différentes déclinaisons du mot «service » reviennent à de nombreuses reprises, en l'espace d'une dizaine de lignes: «lui offrit ses services »; «il le servirait en cela»; «chacun des services ${ }^{49}$. Ces exemples insistent sur le manque, certes illusoire, d'intérêt personnel dans le «commerce» que fait Oxtiern à Sanders, à qui il promet d'établir à Stockholm, même sans la promesse de se marier avec sa

47. Ibid., p. 390.

48. Ibid., p. 376.

49. Ibid., p. 390-391. 
fille. La nouvelle souligne en outre l'inquiétante «durée» des projets de la Scholtz qui prépare lentement son attaque, «Environ trois mois passèrent ainsi, sans que la veuve s'expliquât davantage »; plus loin, «Il $y$ avait environ six mois que les choses étaient en cet état, lorsque le comte Oxtiern [fut] obligé de venir de Stockholm à Nordkoping [...] »; et, enfin, «Quelques semaines passèrent ainsi, lorsqu'on vit arriver dans Nordkoping une voiture superbe accompagnée de plusieurs valets $[\ldots] »^{50}$. Le passage du temps est marqué pour représenter l'illusion de sécurité qu'il accorde aux victimes; loin de signifier la paix, il signifie la gradation de suspense et un certain calme avant la tempête, à savoir la même stratégie des financiers anglais, avides de s'emparer « un jour » des mines suédoises. La dynamique du commerce renvoie à une stratégie mercantiliste où le modèle de la conquête externe contamine la sociabilité de l'échange interne ${ }^{51}$.

\section{L'histoire et la fiction de la mine}

À l'aide des ressources historiques qu'on peut trouver sur les mines suédoises, on peut déduire que la description des rapports anglo-suédois relatés par le marquis est fausse, entièrement de la main de l'auteur. Les mines auxquelles Ernestine fait référence n’ont jamais été sous le contrôle des Anglais. Certes, l'économie suédoise périclite à la fin du XVII ${ }^{\mathrm{e}}$ siècle, situation que le narrateur décrit comme un commerce «flétri » et «dérangé », notamment à cause de l'écroulement de l'industrie du cuivre. Cela n'est pas cependant à cause des dettes contractées avec l'Angleterre; aucun détail dans l'histoire économique de la Suède ne confirme cet élément de la nouvelle. Gabriel Jars explicite à ce propos que «toutes les mines en général, de quelque nature que ce soit, appartiennent à la Couronne sans aucune distinction $^{52}$ ». Par ailleurs, si l'industrie minière en Suède avait été contrôlée par les Anglais, elle ne serait probablement pas restée aussi arriérée

50. Ibid., p. $3^{85}, 388$ et 403 ; je souligne.

51. Selon Lionel Rothkrug (1965) et Catherine Larrère (1992), le mercantilisme peut être considéré comme le «machiavélisme des grands États modernes » : "Cela passe par la relégation de la morale, particulièrement dans les rapports entre États: la pluralité d'États, indépendants et rivaux, définit la situation internationale que n'unifie, ni ne soumet, aucune instance morale supérieure » (Catherine Larrère, L'invention de l'économie au XVIII siècle, Paris, PUF, 1992, p. 96).

52. Gabriel Jars, Voyages métallurgiques, op. cit., t. 1, 8émoire, p. 101. 
qu'elle ne l'a été durant le XVIII ${ }^{\mathrm{e}}$ siècle ${ }^{53}$. En fait, les vraies causes de la dépression économique de la Suède à la fin du règne de Charles XII sont tout autres que celles qu'invente le marquis. En les découvrant, on sera plus à même de rendre raison de cet ajout fictif qui n'est pas sans intérêt pour la compréhension de la nouvelle.

Pour ce faire, il faut enquêter sur les sources historiques, les langages économiques qui sont susceptibles d'inspirer l'écrivain. D'où tire-t-il sa connaissance du pays scandinave? Les nombreuses mentions de Charles XII nous conduisent vers l'ouvrage de Voltaire, son Histoire de Charles XII, roi de Suède, publié à partir de 1731 et qui fait l'objet de plus de 60 rééditions du vivant du philosophe. Dans cet ouvrage, un événement en particulier se distingue de la trame de la vie de Charles XII, qui fait écho à celui relaté par le marquis. À la fin du règne du roi, Voltaire invoque lui aussi une augmentation de dettes qui laisse l'économie de la nation en ruine. Or ces dettes fatidiques ne sont pas celles que la Suède fait avec l'Angleterre; ce sont celles qu'établit la Suède elle-même pour remédier aux extravagances de son histoire «héroïque», celles mises en place par le fameux baron de Görtz, que l'histoire suédoise caractérise à tort comme le véritable auteur du déclin économique du royaume.

Dans l'histoire suédoise du début du XviII siècle, le baron de Görtz est souvent considéré comme une bête noire à cause de son projet d'augmenter la valeur du cuivre pour renflouer la trésorerie de l'État, épuisée par la longue succession de guerres menées par le roi à travers le nord de l'Europe. Ce projet s'est évidemment soldé par l'échec, la totale dévalorisation du cuivre, ainsi que par la dévastation économique du pays. Selon Voltaire,

Le baron de Görtz donna alors une libre étendue à un projet qu'il avait déjà essayé avant d'aller en France et en Hollande; c'était de donner au

53. Eli Heckscher, grand historien de l'économie suédoise, a beaucoup travaillé sur les retards industriels de la Suède à l'époque moderne. Selon lui, la Suède souffrait d'une crainte de déforestation liée à son isolement économique et industriel qui restait sourd aux avancées des Anglais qui, dès le début du siècle, commençaient à se passer du charbon de bois pour lui substituer la fonte au coke. Les Suédois, eux, étaient moins productifs, hésitant à se servir de leur bois à une échelle industrielle, ignorant en plus d'autres matières qui pouvaient servir de combustible (Eli Heckscher, «Un grand chapitre de l'histoire du fer: le monopole suédois», Annales d'histoire économique et sociale, 15, 1932, p. 225-241; et aussi An Economic History of Sweden, Cambridge, Harvard University Press, 1954). 
cuivre la même valeur qu'à l'argent; de sorte qu'une pièce de cuivre, dont la valeur intrinsèque est un demi-sou, passait pour quarante sous avec la marque du prince; à peu près comme dans une ville assiégée les gouverneurs ont souvent payé les soldats et les bourgeois avec de la monnaie de cuir, en attendant qu'on pût avoir des espèces réelles. Ces monnaies fictives, inventées par la nécessité, et auxquelles la bonne foi seule peut donner un crédit durable, sont comme des billets de change, dont la valeur imaginaire peut excéder aisément les fonds qui sont dans un État ${ }^{54}$.

Voltaire est soucieux de mettre en lumière les expériences du baron; le projet d'augmenter la valeur du cuivre fonctionne selon une logique de crédit « qu'il avait déjà essayé ». Görtz n’agit donc pas aveuglément. De plus, bien que s'appuyant sur des ressources «fictives », la stratégie est particulièrement moderne pour l'époque, traitant l'argent moins comme une fin qu'un moyen de stimuler les échanges. Voltaire concède que le crédit s'établit non par fantaisie mais par «nécessité » tout en essayant de rétablir, autant que faire se peut, la postérité de Görtz, ce magistrat qui voulait assez sagement corriger les intempérances couteuses du roi qui est, lui, l'origine première des troubles économiques de la Suède. Si Voltaire ne va pas jusqu'à faire une apologie de Görtz, il critique le fanatisme avec lequel il est condamné, bouc-émissaire d'un peuple qui ne veut pas se désillusionner des chimères de l'héroïsme. «Les peuples, toujours pleins de vénération pour Charles XII, dit-il, n'osaient presque le haïr, et faisaient tomber le poids de leur aversion sur un ministre (Görtz), qui comme étranger, et comme gouvernant les finances, était doublement assuré de la haine publique $^{55}$ ». En cela, l'Histoire de Charles XII raconte les dangers économiques d'une «vertu» trop abondante, d'un héroïsme trop fougueux. Une telle politique porte atteinte au commerce dont la robustesse assure la stabilité d'une nation et la protège, comme le montrera plus tard Hirschman, de la tyrannie:

Sa fermeté, devenue opiniâtreté, fit ses malheurs dans l'Ukraine, et le retint cinq ans en Turquie; sa libéralité, dégénérant en profusion, a ruiné la Suède; son courage, poussé jusqu’à la témérité, a causé sa mort; sa justice a été quelquefois jusqu'à la cruauté, et, dans les dernières

54. Voltaire, Histoire de Charles XII, roi de Suède, édité par Gunnar von Proschwitz, Euvres complètes de Voltaire, Oxford, Voltaire Foundation, 1996, t. 4.

55. Ibid., p. 534. 
années, le maintien de son autorité approchait de la tyrannie. Ses grandes qualités, dont une seule eût pu immortaliser un autre prince, ont fait le malheur de son pays ${ }^{56}$.

Le portrait que dresse Voltaire du grand monarque suédois souligne un courant général d'anti-héroïsme qui réduit la gloire à de l'intempérance et à de l'immodération. Puisque Charles XII réunit trop de vertus, ses nombreuses qualités (fermeté, libéralité, courage, justice) deviennent des sources de malheur, des vertus transformées en vices. La mention de l'«immortalité » des princes infirme, de surcroît, l'intérêt de l'héroïsme qui est de rester dans la mémoire des hommes par la commémoration historique. Aux yeux du philosophe, les sacrifices de Charles XII n'assurent plus sa gloire, car celle-ci requiert un calcul plus prudent. D'où l'image de la rentabilité qu'il utilise pour raisonner la postérité: «Ses grandes qualités, dont une seule eût pu immortaliser un autre prince». La gloire fait dorénavant l'objet d'une économie qui la sape, à savoir la source des ressorts et freins nécessaires pour influencer, voire abaisser, les passions du social.

Dans le cas de Sade, l'histoire de l'économie suédoise, aussi bien que l'épisode de Görtz, présentent un problème dans la mesure où le rôle du crédit n'est pas une attaque anglaise, mais constituent une menace «interne». Même si Görtz était allemand - Voltaire le dit bien -, il était conseiller du roi, ses programmes économiques déterminaient la politique de Charles XII qui ne l'a jamais démenti. L'augmentation de la valeur du cuivre instaurée par Görtz ternit l'histoire nationale et ponctue la postérité de Charles XII par un échec financier qui souligne l'aveuglement d'un monarque relativement à des questions économiques que Voltaire juge plus importantes pour la société que les guerres et les conquêtes. En remplaçant Görtz par les Anglais, la fiction de la nouvelle rend possible l'extériorisation de la menace financière et présente l'embourgeoisement de la société comme une influence exogène (qu'on songe encore à Alaric, Attila et Théodoric) qui n’est pas inhérente à l'identité suédoise, incarnée par Sanders, entièrement réhabilitée à la fin du récit. La représentation du commerce comme une influence étrangère rend plus vraisemblable la résurrection de la vertu en même temps qu'elle purifie le mythe

56. Ibid., p. 542 . 
d'héroïsme considérablement dénigré par un libéralisme économique d'inspiration «anglaise $»^{57}$.

Les mines, avec toute leur civilité fantaisiste, participent d'une rhétorique mythologique qui fournit un espace et une trajectoire verticale aux vicissitudes de la fortune. Les mines, autrefois la grande ressource militaire de la Suède, sont maintenant sous le contrôle d'une nation étrangère; Oxtiern, qui était aux plus hauts rangs de la société, se retrouve au niveau le plus inférieur, dans le souterrain industriel qui rend possible le luxe d'en haut. Ce revirement de la fortune prépare le redressement héroïque qui s'exprime à l'encontre d'un obstacle qui l'opprime. Ainsi, Ernestine accomplit la mission de l'Idée sur les romans qui propose de créer de nouveaux «dieux", voire une nouvelle forme de religion tout aussi «superstitieuse» que l'adhésion à une Cité céleste, mais plus « raisonnable» en raison de son caractère civil. Dans les études les plus conséquentes qui portent sur le rapport entre Sade et la religion ${ }^{58}$, on n'a pas assez souligné la dimension sacrale du romanesque, dont la raison d'être ne vise pas la vérité mais l'immortalisation des libérateurs, tel Hercule, ou des personnages qui lui «ressemblent», comme Sanders: «Hercule, grand capitaine, dut vaillamment combattre ses ennemis; voilà le héros et l'histoire; Hercule détruisant des monstres, pourfendant des géants : voilà le dieu... ${ }^{59}$ » Ainsi, selon Sade, l'écriture romanesque ne cherche pas à nier Dieu, mais à le supplanter par une religion civile. Le marquis continue dans ce sens le projet du

57. On peut aussi comparer la représentation du crédit dans Ernestine avec la lettre X sur le commerce de Voltaire où le crédit permet aux Italiens de s'acheter des armes pour se battre contre l'armée royale: "Quand Louis XIV faisait trembler l'Italie, et que ses armées déjà maitresses de la Savoie et du piémont étaient prêtes de prendre Turin, il fallut que le prince Eugène marchât du fond de l'Allemagne au secours du duc de Savoie; il n'avait point d'argent, sans quoi on ne prend ni ne défend des villes; il eut recours à des marchands anglais; en une demi-heure de temps, on lui prêta cinquante millions. Avec cela il délivra Turin, battit les Français, et écrivit à ceux qui avaient prêté cette somme ce petit billet: "Messieurs, j’ai reçu votre argent et je me flatte de l'avoir employé à votre satisfaction” » ("Lettre X», Lettres philosophiques, édité par Olivier Ferret et Antony McKenna, Paris, Classiques Garnier, 2010, p. 97).

58. Voir le deuxième livre que Jeangène Vilmer consacre à Sade, La religion de Sade, Paris, Atelier, 2008; voir aussi les études de Muriel Schmid, Le souffre au bord de la chaire: Sade et l'évangile, Genève, Labor et Fides, 2001; et «The Sacrament of Penance in Sade's Writings: A Practice between Hell and Apathy », Eighteenth Century Fiction, XV, 3-4, 2003, p. 761-778.

59. Idée sur les romans, op. cit., p. 62-63. 
dernier chapitre du Contrat social de Rousseau, mais en lui enlevant son ambiguïté, c'est-à-dire son esthétique de l'impasse et de la résignation, si caractéristique de l'écriture politique du Genevois ${ }^{60}$. La «raison » superstitieuse fonctionne selon une sorte d'oxymore qu'on a intérêt à comprendre du point de vue d'une économie de la gloire ou de l'immortalité héroïque. Selon R. Morrissey, la « raison de la gloire» est une raison différente de celle qui guide le «présent », où la morale a pour principe l'utilité et l'amour de soi. La gloire, que Sade appelle la «reconnaissance due aux libérateurs», devient une «monnaie morale» qui libère l'homme d'une motivation immanente ainsi que de ses intérêts particuliers. Elle permet à celui-ci d'accéder à une «transcendance dans l'immanence» fondée sur l'imaginaire et la fiction. Selon R. Morrissey,

Pour dégager [l'homme] de l'immédiateté, des contingences du moment et de l'appel des appétits comme des instincts, l'idée de la transcendance dans l'immanence joue un rôle capital. Car la pensée de la gloire permet à l'homme trop rudoyé par le présent d'imaginer, par la projection de sa propre estime pour ceux qui l'ont précédé, la considération, les hommages des hommes à venir. C'est en sortant du présent, par une projection de soi dans le temps, que l'homme accède à une grandeur morale et à une rationalité différente de celle du présent ${ }^{61}$.

La «raison » de la gloire est autant un rejet du présent-réel qu’une dialectique du présent-réel avec le «futur», moins une temporalité qu’une récompense imaginaire qui motive la personne héroïque. Elle fonctionne selon une logique de la rétribution et de la récompense qui remplace la bassesse des motifs pécuniaires ou religieux. La gloire qu'on envisage à la fin du XVIII ${ }^{\mathrm{e}}$ siècle est grandement corrective et réunificatrice, un moyen terme qui négocie la fracture entre la vertu

6o. À la fin du chapitre «De la religion civile» du Contrat social, Rousseau admet que la religion civile est nécessaire pour une république, mais qu’aucune religion existante n'est à même de s'acquitter de la fonction politique; le christianisme ni ses alternatives ne s'avèrent adéquats (voir sur ce point, Robert Beiner, Civil Religion: A Dialogue in the History of Political Philosophy, New York / Cambridge, Cambridge University Press, 2011, p. 78-86). Enfin, le chapitre pose plus de problèmes qu'il n'en résout, tout comme le texte dans son ensemble, qui semble exclure les idées qu'il propose d'un projet activiste, stratégie discursive que Judith Shklar a décrite comme une "esthétique de la résignation" (Men and Citizens: A Study of Rousseau's Social Theory, Cambridge, Cambridge University Press, 1969, p. 30).

61. Robert Morrissey, Napoléon et l'héritage de la gloire, op. cit., p. 83-84. 
et le commerce, comme le fait Napoléon, entre les Anciens et les Modernes.

Face à l'histoire économique de la Suède, la mine d'Ernestine est certes transformée par l'écriture. La mission romanesque qui repose pour Sade sur un mélange de la réalité et de la fiction superpose différents niveaux de représentation. Si les références spatiotemporelles déterminent un effet de réalité qui se neutralise face au merveilleux de la description, l'explication de la propriété des mines, elle, est plus fourbe. La falsification «historique» sert à harmoniser la juxtaposition entre la réalité et la fiction, processus de fonte qui est nécessaire pour la construction du mythe. C'est pourquoi il ne faut pas oublier la force transformative de la mine à l'intérieur du récit qui tend à influer sur la corruption des personnages de l'intrigue. Oxtiern et même Sanders en sortent améliorés, moralement intègres. La mine fait plonger ses visiteurs au fond des vicissitudes de la fortune pour ensuite devenir le lieu où un nouvel héroïsme peut s'affirmer; la véritable «civilité» de la mine consiste à convertir des passions corrompues en des forces morales qui remontent ensuite à la surface. La fiction opère ainsi une sorte d'investissement moral: en y bannissant un criminel, la société en reçoit deux héros; un taux de rendement plutôt compétitif. 